\title{
Measurement of a Quantum System Coupled to Independent Heat-Bath and Pointer Modes
}

\author{
Dima Mozyrsky and Vladimir Privman \\ Department of Physics, Clarkson University \\ Potsdam, New York 13699-5820, USA \\ Electronic mail: privman@clarkson.edu
}

To be published in Modern Physics Letters B (2000). 


\begin{abstract}
We present an exact derivation of a process in which a microscopic measured system interacts with heat-bath and pointer modes of a measuring device, via a coupling involving a general Hermitian operator $\Lambda$ of the system. In the limit of strong interaction with these modes, over a small time interval, we derive the exact effective many-body density matrix of the measured system plus pointer. We then discuss the interpretation of the dynamics considered as the first stage in the process of quantum measurement, eventually involving the wave-function collapse due to interactions with "the rest of the universe." We establish that the effective density matrix represents the required framework for the measured system and the pointer part of the measuring device to evolve into a statistical mixture described by direct-product states such that the system is in each eigenstate of $\Lambda$ with the correct quantum-mechanical probability, whereas the expectation values of pointer-space operators retain amplified information of the system's eigenstate.
\end{abstract}


The problem of quantum measurement has fascinated scientists for a long time $[1,2]$. It has been argued that a large "bath" is an essential ingredient of the measurement process. Interaction with the bath, which might be a heat-bath in thermal equilibrium, causes decoherence which is needed to form a statistical mixture of eigenstates out of the initially fully or partially coherent quantum state of the measured system. An "external" bath ("the rest of the universe") may also play a role in selection of those quantum states of the pointer that manifest themselves in classical observations [2-7]. In this work we propose a model in which the pointer retains information on the measurement result because of its coupling to the measured system, without the need to couple it also to the internal bath. The measured system is still coupled to the internal bath.

In an exactly solvable model of a quantum oscillator coupled to a heat bath of oscillators, it has been shown [4] that the reduced density matrix of the system, with the bath traced over, decoheres, i.e., it looses its offdiagonal elements in the eigenbasis of the interaction Hamiltonian. Recent work on decoherence [8-11] has explored the latter effect for rather general cases, for bosonic (oscillator) and spin baths. Applications for various physical systems have been reported [12-18]. Fermionic heat bath has also been used in the literature [19].

It is clear, however, that the full function of a large, multimode measuring device, interacting with a small (microscopic) quantum system, must 
be different from thermal equilibration or similar averaging effect. The device must store and amplify the measurement outcome information. In this work we propose a solvable model that shows how this is accomplished.

It must be stressed that for a complete description of the measurement process one needs to interpret the transfer of the information stored after the system-pointer and system-internal bath interaction to the macroscopic level [2]. Our attention here is no the process which corresponds to the first stage of the measurement, in which the pointer acquires amplified information by entanglement with the state of the system. Thus we do not claim to resolve the foundation-of-quantum mechanics issue of how that information is passed on to the classical world, involving the collapse of the wave functions of the system and each pointer mode. Indeed, it is unlikely that the wave function collapse can be fully described within the quantummechanical description of the three subsystems involved. Presumably, it would require consideration of an external bath with which the pointer and the internal bath interact. This problem is not presently solved [1-3], and we first sidestep it by assuming separation of time scales (see below). However, we later argue that our results provide useful hints on how to view the larger problem of quantum measurement.

We now identify the three quantum systems involved. First, the measured system, $S$, is a microscopic system with the Hamiltonian which will be also denoted by $S$. Second, the measuring device must have the "bath" 
or "body" part, $B$, containing many individual modes. The $k$ th mode will have the Hamiltonian $B_{k}$. The bath part of the device is not observed, i.e., it can be traced over. Finally, the device must also have modes that are not traced over. These modes constitute the pointer, $P$, that amplifies the information obtained in the measurement process and can later pass it on for further amplification or directly to macroscopic (classical) systems. The $m$ th pointer mode has the Hamiltonian $P_{m}$. It is expected that expectation values of some quantities in the pointer undergo a large change during the measurement process.

It turns out, a posteriori, that the device modes involved in the measurement process can be quite simple, and they need not interact with each other. This assumption allows us to focus on the evolution of the system $S$ and its effect on the pointer $P$. However, it is the pointer's interaction with the external bath (some external modes, "the rest of the universe") that is presumed to select those quantum states of $P$ that manifest themselves classically. For now, let us avoid the discussion of this matter, see [2-6], by assuming that the added evolution of the pointer due to such external interactions occurs on time scales larger than the measurement time, t. Similarly, when we state that the internal bath modes can be "traced over," we really mean that their interactions with the rest of the universe are such that these modes play no role in the wave-function-collapse stage of the measurement process. 
Furthermore, the measurement process probes the wavefunction of the measured system at the initial time, $t=0$, rather than its time evolution under $S$ alone. It is ideally instantaneous. In practice, it is faster than the time scales associated with the dynamics under $S$. Such a process can be obtained as the limit of a system in which very strong interactions between $S$ and $B$, and also between $S$ and $P$, are switched on at $t=0$ and switched off at $t>0$, with small time interval $t$. At later times, the pointer can interact with other, external systems to pass on the result of the measurement.

Thus, we assume that the Hamiltonian of the system itself, $S$, can be ignored in the process. The total Hamiltonian of the system plus device will be taken as

$$
H=\sum_{k} B_{k}+\sum_{m} P_{m}+b \Lambda \sum_{k} L_{k}+p \Lambda \sum_{m} C_{m}
$$

Here $\Lambda$ is some Hermitian operator of the system that couples to certain operators of the modes, $L_{k}$ and $C_{m}$. The parameters $b$ and $p$ are introduced to measure the coupling strength for the bath and pointer modes, respectively. They are assumed very large; the ideal measurement process corresponds to $b, p \rightarrow \infty$.

We note that the modes of $P$ and $B$ can be similar. The only difference between the bath and pointer modes is in how they interact with the "rest of the universe": the bath is traced over (unobserved), whereas the pointer modes have their wave functions collapsed in a later step of the measurement process. Thus, we actually took the same coupling operator $\Lambda$ for the bath 
and pointer. In fact, all the exact calculations reported in this work can be also carried out for different coupling operators $\Lambda_{b}$ and $\Lambda_{p}$, for the bath and pointer modes, provided they commute, $\left[\Lambda_{b}, \Lambda_{p}\right]=0$, so that they share a common set of eigenfunctions. The final wavefunction of the measured system, after the measurement, is in this set. Analytical calculation can be even extended to the case when the system's Hamiltonian $S$ is retained in (1), provided all three operators, $S, \Lambda_{b}, \Lambda_{p}$, commute pairwise. The essential physical ingredients of the model are captured by the simpler choice (1).

We will later specify all the operators in (1) as the modes of the bosonic heat bath of Caldeira-Leggett type $[17,19-26]$. For now, however, let us keep our discussion general. We will assume that the system operator $\Lambda$ has nondegenerate, discrete spectrum of eigenstates:

$$
\Lambda|\lambda\rangle=\lambda|\lambda\rangle
$$

Some additional assumptions on the spectrum of $\Lambda$ and $S$ will be encountered later. We also note that the requirement that the coupling parameters $b$ and $p$ are large may in practice be satisfied because, at the time of the measurement, the system's Hamiltonian $S$ corresponds to slow or trivial dynamics.

Initially, at $t=0$, the quantum systems $(S, B, P)$ and their modes are not correlated with each other. We assume that $\rho$ is the initial density matrix of the measured system. The initial state of each bath and pointer mode 
will be assumed thermalized, with $\beta=1 /(k T)$ and the density matrices

$$
\theta_{k}=\frac{e^{-\beta B_{k}}}{\operatorname{Tr}_{k}\left(e^{-\beta B_{k}}\right)} \quad \sigma_{m}=\frac{e^{-\beta P_{m}}}{\operatorname{Tr}_{m}\left(e^{-\beta P_{m}}\right)}
$$

We cannot offer any fundamental physical reason for having the initial bath and pointer mode states thermalized, especially for the pointer; this choice is made to allow exact solvability.

The density matrix of the system at the time $t$ is

$$
R=e^{-i H t / \hbar} \rho\left(\prod_{k} \theta_{k}\right)\left(\prod_{m} \sigma_{m}\right) e^{i H t / \hbar}
$$

The bath is not probed and it can be traced over. The resulting reduced density matrix $r$ of the combined system $S+P$ will be represented by its matrix elements in the eigenbasis of $\Lambda$. These quantities are each an operator in the space of $P$ :

$$
r_{\lambda \lambda^{\prime}}=\left\langle\lambda\left|\operatorname{Tr}_{B}(R)\right| \lambda^{\prime}\right\rangle
$$

We now assume that operators in different spaces and of different modes commute. Then one can show that

$$
\begin{gathered}
r_{\lambda \lambda^{\prime}}=\rho_{\lambda \lambda^{\prime}}\left[\prod_{m} e^{-i t\left(P_{m}+p \lambda C_{m}\right) / \hbar} \sigma_{m} e^{i t\left(P_{m}+p \lambda^{\prime} C_{m}\right) / \hbar}\right] \times \\
{\left[\prod_{k} \operatorname{Tr}_{k}\left\{e^{-i t\left(B_{k}+b \lambda L_{k}\right) / \hbar} \theta_{k} e^{i t\left(B_{k}+b \lambda^{\prime} L_{k}\right) / \hbar}\right\}\right]}
\end{gathered}
$$

where $\rho_{\lambda \lambda^{\prime}}=\left\langle\lambda|\rho| \lambda^{\prime}\right\rangle$. This result involves products of $P$-space operators and traces over $B$-space operators which are all single-mode. Therefore, 
analytical calculations are possible for some choices of the Hamiltonian (1). The observable $\Lambda$ can be kept general.

The role of the product of traces over the modes of the bath in (6) is to induce decoherence which is recognized as essential for the measurement process, e.g., $[1,2]$. At the time $t$, the absolute value of this product should approach $\delta_{\lambda \lambda^{\prime}}$ in the limit of large $b$. Let us now assume that the bath is bosonic. The Hamiltonian of each mode is then $\hbar \omega_{k} a_{k}^{\dagger} a_{k}$, where for simplicity we shifted the zero of the oscillator energy to the ground state. The coupling operator $L_{k}$ is usually selected as $L_{k}=g_{k}^{*} a_{k}+g_{k} a_{k}^{\dagger}$. For simplicity, though, we will assume that the coefficients $g_{k}$ are real:

$$
B_{k}=\hbar \omega_{k} a_{k}^{\dagger} a_{k} \quad L_{k}=g_{k}\left(a_{k}+a_{k}^{\dagger}\right)
$$

For example, for radiation field in a unit volume, coupled to an atom [27], the coupling is via a linear combination of the operators $\left(a_{k}+a_{k}^{\dagger}\right) / \sqrt{\omega_{k}}$ and $i\left(a_{k}-a_{k}^{\dagger}\right) / \sqrt{\omega_{k}}$. For a spatial oscillator, these are proportional to position and momentum, respectively. Our calculations can be extended to have an imaginary part of $g_{k}$ which adds interaction with momentum.

The product of traces in (6) can be calculated by coherent-state or operator-identity techniques [8-10]. Here and below we only list the results of such calculations which are usually quite cumbersome:

$$
\begin{gathered}
\prod_{k} \operatorname{Tr}_{k}\{\ldots\}=\exp \left\{-2 b^{2}\left(\lambda-\lambda^{\prime}\right)^{2} \Gamma(t)+i b^{2}\left[\lambda^{2}-\left(\lambda^{\prime}\right)^{2}\right] \gamma(t)\right\} \\
\Gamma(t)=\sum_{k}\left(\hbar \omega_{k}\right)^{-2} g_{k}^{2} \sin ^{2} \frac{\omega_{k} t}{2} \operatorname{coth} \frac{\hbar \beta \omega_{k}}{2}
\end{gathered}
$$


Explicit form of $\gamma(t)$ is also known [8].

In the continuum limit of many modes, the density of the bosonic bath states in unit volume, $D(\omega)$, and the Debye cutoff with frequency, $\omega_{D}$, are introduced [22] to get

$$
\Gamma(t)=\int_{0}^{\infty} d \omega \frac{D(\omega) g^{2}(\omega)}{(\hbar \omega)^{2}} e^{-\omega / \omega_{D}} \sin ^{2} \frac{\omega t}{2} \operatorname{coth} \frac{\hbar \beta \omega}{2}
$$

Let us consider the popular choice termed Ohmic dissipation [22], motivated by atomic-physics [27] and solid-state applications [22], corresponding to

$$
D(\omega) g^{2}(\omega)=\Omega \omega
$$

where $\Omega$ is a constant. Other powers of $\omega$ have also been considered, e.g., [11]. In studies of decoherence [8-11] for large times $t$, for models without strong coupling, not all the choices of $D(\omega) g^{2}(\omega)$ lead to complete decoherence [11] because $\Gamma(t)$ must actually diverge to $+\infty$ for $t \gg \hbar \beta$, as happens for the choice (11).

Let us assume that the energy gaps of $S$ are bounded so that there exists a well defined time scale $\hbar / \Delta S$ of the evolution of the system under $S$. There is also the time scale $1 / \omega_{D}$ set by the frequency cutoff assumed for the interactions. The thermal time scale is $\hbar \beta$. The only real limitation on the duration of measurement is that $t$ must be less then $\hbar / \Delta S$. In applications, typically [22] one can assume that $1 / \omega_{D} \ll \hbar / \Delta S$. Furthermore, it is customary to assume that the temperature is low [22],

$$
\begin{gathered}
t \text { and } 1 / \omega_{D} \ll \hbar / \Delta S \ll \hbar \beta \\
-10-
\end{gathered}
$$


In the limit of large $\hbar \beta$, the absolute value of (8) reduces to

$$
\operatorname{Abs}\left(\prod_{k} \operatorname{Tr}_{k}\{\ldots\}\right) \simeq \exp \left\{-\frac{\Omega}{2 \hbar^{2}} b^{2}\left(\lambda-\lambda^{\prime}\right)^{2} \ln \left[1+\left(\omega_{D} t\right)^{2}\right]\right\}
$$

In order to achieve effective decoherence, the product $(\Delta \lambda)^{2} b^{2} \ln \left[1+\left(\omega_{D} t\right)^{2}\right]$ must be large. The present approach only applies to operators $\Lambda$ with nonzero scale of the smallest spectral gaps, $\Delta \lambda$.

We note that the decoherence property needed for the measurement process will be obtained for nearly any well-behaved choice of $D(\omega) g^{2}(\omega)$ because we can rely on the value of $b$ being large rather than on the properties of the function $\Gamma(t)$. If $b$ can be large enough, very short measurement times are possible. However, it may be advisable to use measurement times $1 / \omega_{D} \ll t \ll \hbar / \Delta S$ to get the extra amplification factor $\sim \ln \left(\omega_{D} t\right)$ and allow for fuller decoherence and less sensitivity to the value of $t$ in the pointer part of the dynamics, to be addressed shortly. We notice, furthermore, that the assumption of a large number of modes is important for monotonic decay of the absolute value of (8) in decoherence studies [8-11], where irreversibility is obtained only in the limit of infinite number of modes. In our case, it can be shown that such a continuum limit allows to extend the possible measurement times from $t \ll 1 / \omega_{D}$ to $1 / \omega_{D} \ll t \ll \hbar / \Delta S$.

Consider the reduced density matrix $r$ of $S+P$, see (6). It becomes diagonal in $|\lambda\rangle$, at the time $t$, because all the nondiagonal elements are 
small,

$$
r=\sum_{\lambda}|\lambda\rangle\langle\lambda| \rho_{\lambda \lambda} \prod_{m} e^{-i t\left(P_{m}+p \lambda C_{m}\right) / \hbar} \sigma_{m} e^{i t\left(P_{m}+p \lambda C_{m}\right) / \hbar}
$$

Thus, the described stage of the measurement process yields the density matrix that can be interpreted as describing a statistically distributed system, without quantum correlations. This, however, is only meaningful within the ensemble interpretation of quantum mechanics.

For a single system plus device, coupling to the rest of the universe is presumably needed (this problem is not fully understood in our opinion, see [2]) for that system to be left in one of the eigenstates $|\lambda\rangle$, with probability $\rho_{\lambda \lambda}$. After the measurement interaction is switched off at $t$, the pointer coupled to that system will carry information on the value of $\lambda$. This information is "amplified," owing to the large parameter $p$ in the interaction.

We note that one of the roles of the pointer having many modes, many of which can be identical and noninteracting, is to allow it (the pointer only) to still be treated in the ensemble, density matrix description, even if we focus on the later stages of the measurement when the wave functions of a single measured system and of each pointer mode are already collapsed. This pointer density matrix can be read off (14). This aspect is new and it may provide a useful hint on how to set up the treatment of the full quantum-measurement process description.

Another such hint is provided by the fact that, as will be shown shortly, the changes in the expectation values of some observables of the pointer re- 
tain amplified information on the system's eigenstate. So, coupling to the rest of the universe that leads to the completion of the measurement process, should involve such an observable of the pointer. Eventually, the information in the pointer, perhaps after several steps of amplification, should be available for probe by interactions with classical devices.

At time $t=0$, expectation values of various operators of the pointer will have their initial values. These values will be different at the time $t$ of the measurement owing to the interaction with the measured system. It is expected that the large coupling parameter $p$ will yield large changes in expectation values of the pointer quantities. This does not apply equally to all operators in the $P$-space. Let us begin with the simplest choice: the Hamiltonian $\sum_{m} P_{m}$ of the pointer.

We will assume that the pointer is described by the bosonic heat bath and, for simplicity, use the same notation for the pointer modes as that used for the bath modes. The assumption that the pointer modes are initially thermalized, see (3), was not used thus far. While it allows exact analytical calculations, it is not essential: the effective density matrix describing the pointer modes at the time $t$, for the system's state $\lambda$, will retain amplified information on the value of $\lambda$ for general initial states of the pointer.

This effective density matrix is the product over the $P$-modes in (14). For the "thermal" $\sigma_{m}$ from (3), the expectation value of the pointer energy 
$E_{P}$ can be calculated from

$$
\begin{gathered}
\left\langle E_{P}\right\rangle_{\lambda} \operatorname{Tr}_{P}\left(e^{-\hbar \beta \sum_{s} \omega_{s} a_{s}^{\dagger} a_{s}}\right)=\operatorname{Tr}_{P}\left\{\left(\sum_{m} \hbar \omega_{m} a_{m}^{\dagger} a_{m}\right) \times\right. \\
\left.\prod_{n}\left[e^{-i t\left[\omega_{n} a_{n}^{\dagger} a_{n}+p \lambda g_{n}\left(a_{n}+a_{n}^{\dagger}\right)\right] / \hbar}\left(e^{-\hbar \beta \sum_{k} \omega_{k} a_{k}^{\dagger} a_{k}}\right) e^{i t\left[\omega_{n} a_{n}^{\dagger} a_{n}+p \lambda g_{n}\left(a_{n}+a_{n}^{\dagger}\right)\right] / \hbar}\right]\right\} .
\end{gathered}
$$

The right-hand side can be reduced to calculations for individual modes. Operator identities can be then utilized to obtain the results

$$
\begin{gathered}
\left\langle E_{P}\right\rangle_{\lambda}(t)=\left\langle E_{P}\right\rangle(0)+\left\langle\Delta E_{P}\right\rangle_{\lambda}(t) \\
\left\langle E_{P}\right\rangle(0)=\hbar \sum_{m} \omega_{m} e^{-\hbar \beta \omega_{m}}\left(1-e^{-\hbar \beta \omega_{m}}\right)^{-2} \\
\left\langle\Delta E_{P}\right\rangle_{\lambda}(t)=\frac{4 p^{2} \lambda^{2}}{\hbar} \sum_{m} \frac{g_{m}^{2}}{\omega_{m}} \sin ^{2}\left(\frac{\omega_{m} t}{2}\right)
\end{gathered}
$$

For a model with Ohmic dissipation, the resulting integral, in the continuum limit, can be calculated to yield

$$
\left\langle\Delta E_{P}\right\rangle_{\lambda}(t)=\frac{2 \Omega \omega_{D} \lambda^{2} p^{2}}{\hbar} \frac{\left(\omega_{D} t\right)^{2}}{1+\left(\omega_{D} t\right)^{2}}
$$

which should be compared to the exponent in (13). The energy will be an indicator of the amplified value of the square of $\lambda$, provided $p$ is large. Furthermore, we see here the advantage of larger measurement times, $t \gg$ $1 / \omega_{D}$. The change in the energy then reaches saturation. After the time $t$, when the interaction is switched off, the energy of the pointer will be conserved. 
Let us consider the expectation value of the following Hermitian operator of the pointer:

$$
X=\sum_{m} C_{m}=\sum_{m} g_{m}\left(a_{m}+a_{m}^{\dagger}\right)
$$

For an atom in a field, $X$ is related to the electromagnetic field operators [24]. One can show that $\left\langle X_{P}\right\rangle(0)=0$ and

$$
\begin{gathered}
\left\langle\Delta X_{P}\right\rangle_{\lambda}(t)=\left\langle X_{P}\right\rangle_{\lambda}(t)=-\frac{4 p \lambda}{\hbar} \sum_{m} \frac{g_{m}^{2}}{\omega_{m}} \sin ^{2}\left(\frac{\omega_{m} t}{2}\right) \\
=-\frac{2 \Omega \omega_{D} \lambda p}{\hbar} \frac{\left(\omega_{D} t\right)^{2}}{1+\left(\omega_{D} t\right)^{2}}
\end{gathered}
$$

The change in the expectation value of $X$ is linear in $\lambda$. However, this operator is not conserved. One can show that after the time $t$ its expectation value decays to zero for times $t+\mathcal{O}\left(1 / \omega_{D}\right)$.

We note that by referring to "unit volume" we have avoided the discussion of the "extensivity" of various quantities. For example, the initial energy $\left\langle E_{P}\right\rangle(0)$ is obviously proportional to the system volume, $V$. However, the change $\left\langle\Delta E_{P}\right\rangle_{\lambda}(t)$ will not be extensive; typically, $g^{2}(\omega) \propto 1 / V$, $D(\omega) \propto V$. Thus, while the amplification in our measurement process can involve a numerically large factor, the changes in the quantities of the pointer will be multiples of microscopic values. Multi-stage amplification, or huge coupling parameter $p$, would be needed for the information in the pointer to become truly "extensive" macroscopically. 
In practice, there will be probably two types of pointer involved in a multistage measurement process. Some pointers will consist of many noninteracting modes. These pointers carry the information, stored in a density matrix rather than a wave function of a single system. The latter transference hopefully makes the wavefunction collapse and transfer of the stored information to the macroscopic level less "mysterious and traumatic." The second type of pointer will involve strongly interacting modes and play the role of an amplifier by utilizing the many-body collective behavior of the coupled modes (phase-transition style). Its role will be to alleviate the artificial requirement for large mode-to-system coupling parameters encountered in our model.

In summary, we described the first stage of a measurement process. It involves decoherence due to a bath and transfer of information to a large system (pointer) via strong interaction over a short period of time. The pointer itself need not be coupled to the internal bath. While we do not offer a solution to the foundation-of-quantum-mechanics wave-function collapse problem [2], our results do provide two interesting observations.

Firstly, the pointer operator "probed" by the rest of the universe during the wave-function collapse stage, may be in part determined not only by how the pointer modes are coupled to the external bath [3-7], but also by the amplification capacity of that operator in the first stage of the process, as illustrated by our calculations. 
Secondly, for a single system (rather then an ensemble), the multiplicity of the (noninteracting) pointer modes might allow the pointer to be treated within the density matrix formalism even after the system and each pointermode wave functions were collapsed. Since it is the information in the pointer that is passed on, this observation might seem to resolve part of the measurement puzzle. Specifically, it might suggest why only those density matrices entering (14) are selected for the pointer: they carry classical (large, different from other values) information in expectation values, rather than quantum-mechanical superposition. However, presumably [2] only a full description of the interaction of the external world with the system $S+P$ can explain the wavefunction collapse of $S$.

We acknowledge helpful discussions with Professor L. S. Schulman. This research has been supported by the US Army Research Office under grant DAAD 19-99-1-0342. 


\section{References}

1. For a historical overview, see, A. Whitaker, Einstein, Bohr and the Quantum Dilemma (Cambridge Univ. Press, Cambridge, 1996).

2. J. Bell, Phys. World 3, August 1990, No. 8, p. 33.

3. W. H. Zurek, Physics Today, October 1991, p. 36.

4. W. G. Unruh, W. H. Zurek, Phys. Rev. D 40, 1071 (1989).

5. W. H. Zurek, S. Habib and J. P. Paz, Phys. Rev. Lett. 70, 1187 (1993).

6. M. Gell-Mann and J. B. Hartle, in Proceedings of the 25th International Conference on High Energy Physics (South East Asia Theor. Phys. Assoc., Phys. Soc. of Japan, Teaneck, NJ, 1991) Vol. 2, p. 1303.

7. M. Gell-Mann and J. B. Hartle, in Quantum Classical Correspondence: The 4th Drexel Symposium on Quantum Nonintegrability, ed. by D. H. Feng and B. L. Hu (International Press, Cambridge, MA, 1997) p. 3.

8. D. Mozyrsky and V. Privman, J. Stat. Phys. 91, 787 (1998).

9. N. G. van Kampen, J. Stat. Phys. 78, 299 (1995).

10. J. Shao, M.-L. Ge and H. Cheng, Phys. Rev. E 53, 1243 (1996).

11. G. M. Palma, K. A. Suominen and A. K. Ekert, Proc. Royal Soc. London A 452, 567 (1996).

12. I. S. Tupitsyn, N. V. Prokof'ev, P. C. E. Stamp, Int. J. Modern Phys. B 11, 2901 (1997).

13. C. W. Gardiner, Handbook of Stochastic Methods for Physics, Chem- 
istry and the Natural Sciences (Springer-Verlag, Berlin, 1990).

14. A. J. Leggett, in Percolation, Localization and Superconductivity, NATO ASI Series B: Physics, ed. by A. M. Goldman and S. A. Wolf (Plenum, NY, 1984), Vol. 109, p. 1.

15. J. P. Sethna, Phys. Rev. B 24, 698 (1981).

16. Review: A. O. Caldeira and A. J. Leggett, Ann. Phys. 149, 374 (1983).

17. A. Garg, Phys. Rev. Lett. 77, 764 (1996).

18. L. Mandel and E. Wolf, Optical Coherence and Quantum Optics (Cambridge Univ. Press, Cambridge, 1995).

19. L.-D. Chang and S. Chakravarty, Phys. Rev. B 31, 154 (1985).

20. A. O. Caldeira and A. J. Leggett, Phys. Rev. Lett. 46, 211 (1981).

21. S. Chakravarty and A. J. Leggett, Phys. Rev. Lett. 52, 5 (1984).

22. Review: A. J. Leggett, S. Chakravarty, A. T. Dorsey, M. P. A. Fisher and W. Zwerger, Rev. Mod. Phys. 59, 1 (1987) [Erratum ibid. 67, 725 (1995)].

23. A. O. Caldeira and A. J. Leggett, Physica 121A, 587 (1983).

24. R. P. Feynman and A. R. Hibbs, Quantum Mechanics and Path Integrals (McGraw-Hill Book Co., NY, 1965).

25. G. W. Ford, M. Kac and P. Mazur, J. Math. Phys. 6, 504 (1965).

26. A. J. Bray and M. A. Moore, Phys. Rev. Lett. 49, 1546 (1982).

27. W. H. Louisell, Quantum Statistical Properties of Radiation (Wiley, NY, 1973). 